\title{
Duoquest: A Dual-Specification System for Expressive SQL Queries
}

\author{
Christopher Baik \\ cjbaik@umich.edu \\ University of Michigan \\ Ann Arbor, MI, USA
}

\author{
Zhongjun Jin \\ markjin@umich.edu \\ University of Michigan \\ Ann Arbor, MI, USA
}

\author{
Michael Cafarella \\ michjc@umich.edu \\ University of Michigan \\ Ann Arbor, MI, USA
}

\author{
H. V. Jagadish \\ jag@umich.edu \\ University of Michigan \\ Ann Arbor, MI, USA
}

\begin{abstract}
Querying a relational database is difficult because it requires users to know both the SQL language and be familiar with the schema. On the other hand, many users possess enough domain familiarity or expertise to describe their desired queries by alternative means. For such users, two major alternatives to writing SQL are natural language interfaces (NLIs) and programming-by-example (PBE). Both of these alternatives face certain pitfalls: natural language queries (NLQs) are often ambiguous, even for human interpreters, while current $\mathrm{PBE}$ approaches require either low-complexity queries, user schema knowledge, exact example tuples from the user, or a closed-world assumption to be tractable. Consequently, we propose dual-specification query synthesis, which consumes both a NLQ and an optional PBE-like table sketch query that enables users to express varied levels of domain-specific knowledge. We introduce the novel dual-specification DuoQUEST system, which leverages guided partial query enumeration to efficiently explore the space of possible queries. We present results from user studies in which Duoguest demonstrates a $62.5 \%$ absolute increase in query construction accuracy over a state-of-the-art NLI and comparable accuracy to a PBE system on a more limited workload supported by the PBE system. In a simulation study on the prominent Spider benchmark, Duoguest demonstrates a $>2 \mathrm{x}$ increase in top-1 accuracy over both NLI and PBE.
\end{abstract}

\section{INTRODUCTION}

Querying a relational database is difficult because it requires users to know both the SQL language and be familiar with the schema. On the other hand, many users possess enough domain familiarity or expertise to describe their desired queries by alternative means. Consequently, an ongoing research challenge is enabling users with domain-specific knowledge but little to no programming background to specify queries.

One popular approach is the natural language interface (NLI), where users can state queries in their native language. Unfortunately, existing NLIs require significant overhead in adapting to new domains and databases $[12,15,22]$ or are overly reliant on specific sentence structures [8]. More recent advances leverage deep learning in an attempt to circumvent

\begin{tabular}{|c|c|c|c|c|c|c|c|}
\hline \multirow[b]{2}{*}{ System } & \multirow[b]{2}{*}{ Soundness } & \multicolumn{3}{|c|}{ Query Expr. ${ }^{1}$} & \multicolumn{3}{|c|}{ Knowledge $^{2}$} \\
\hline & & $\triangleright 4$ & $\sigma$ & $\gamma$ & NS & PT & OW \\
\hline$N L I s[8,22,23]$ & & $\checkmark$ & $\checkmark$ & $\checkmark$ & $\checkmark$ & N/A & $\mathrm{N} / \mathrm{A}$ \\
\hline \multicolumn{8}{|l|}{ PBE Systems } \\
\hline QBE [25] & $\checkmark$ & $\checkmark$ & $\checkmark$ & $\checkmark$ & & $\checkmark$ & $\checkmark$ \\
\hline MWeaver [14] & $\checkmark$ & $\checkmark$ & & & $\checkmark$ & & $\checkmark$ \\
\hline S4 [13] & $\checkmark$ & $\checkmark$ & & & $\checkmark$ & $\checkmark$ & $\checkmark$ \\
\hline SQuID [6] & $\checkmark$ & $\checkmark$ & $\checkmark$ & $\mathfrak{S}^{3}$ & $\checkmark$ & & $\checkmark$ \\
\hline TALOS [18] & $\checkmark$ & $\checkmark$ & $\checkmark$ & $\checkmark$ & $\checkmark$ & & \\
\hline QFE [9] & $\checkmark$ & $\checkmark$ & $\checkmark$ & & & & \\
\hline PALEO [11] & $\checkmark$ & & $\checkmark$ & $\checkmark$ & & & \\
\hline Scythe [19] & $\checkmark$ & $\checkmark$ & $\checkmark$ & $\checkmark$ & & & \\
\hline REGAL+ [17] & $\checkmark$ & $\checkmark$ & $\checkmark$ & $\checkmark$ & $\checkmark$ & & \\
\hline Duoguest & $\checkmark$ & $\checkmark$ & $\checkmark$ & $\checkmark$ & $\checkmark$ & $\checkmark$ & $\checkmark$ \\
\hline
\end{tabular}

Table 1: Duoguest vs. NLI/PBE, considering soundness, query expressiveness, and required user knowledge. $A \checkmark$ is desirable in each column.

these challenges, but the state-of-the-art accuracy [23] on established benchmarks falls well short of the desired outcome, which is that NLIs should either interpret the user's query correctly or clearly detect any errors [12].

Another alternative to writing SQL is programming-byexample (PBE), where users must either provide query output examples or example pairs of an input database and the output of the desired query. PBE systems have the advantage of a concrete notion of soundness in that returned candidate queries are guaranteed to satisfy the user's specification, while NLIs, on the other hand, provide no such guarantees.

However, PBE systems must precariously juggle various factors: how much query expressiveness is permitted, whether schema knowledge is required of the user, whether users may provide partial tuples rather than full tuples, and whether an open-or closed-world setting is assumed, where in a closedworld setting, the user is expected to provide a complete result set, while the user may provide a subset of possible returned tuples in an open-world setting.

Table 1 summarizes the capabilities of previous NLI and PBE systems, with respect to three major categories:

\footnotetext{
${ }_{\triangleright \triangleleft}$ : join, $\sigma$ : selection, $\gamma$ : grouping/aggregation

${ }^{2}$ NS: no schema knowledge, PT: partial tuples, OW: open-world assumption

${ }^{3}$ SQuID does not support projected aggregates (i.e. in the SELECT clause).
} 
(1) soundness, which guarantees that results satisfy the user specification;

(2) permitted query expressiveness;

(3) and required user knowledge.

With respect to these factors, an ideal system would: (1) provide soundness guarantees; (2) enable expressive queries with selections, aggregates, and joins; and (3) allow users to provide partial tuples in an open-world setting without schema knowledge. However, previous approaches could not handle the massive search space produced by this scenario and each constrained at least one of the above factors.

Our Approach - While existing approaches only permit users to specify a single type of specification, we observe that PBE specifications and natural language queries (NLQs) are complementary, as PBE specifications contain hard constraints that can substantially prune the search space, while NLQs provide hints on the structure of the desired SQL query, such as selection predicates and the presence of clauses. Therefore, we argue for dual-specification query synthesis, which consumes both a NLQ and an optional PBE-like specification as input. The dual-specification approach does not inhibit users who are only able to provide a single specification, but can help the system more easily triangulate the desired query when users are able to provide both types of specifications.

System Desiderata - There are several goals in developing a dual-specification system.

First, it is crucial that the dual-specification system helps users without schema knowledge, and potentially even without any SQL experience, correctly construct their desired query. Our aim is to develop a system that can help non-technical users with domain knowledge to construct expressive SQL queries without the need to consult technical experts. In addition, for technical users, such a system can be a useful alternative to manually writing SQL, which often requires the need to manually inspect the database schema.

Second, we want to minimize user effort in using the system. Dual-specification interaction should help users more efficiently synthesize queries, especially in contrast to existing single-specification approaches such as NLIs or PBE systems.

Finally, we also want to have our system run efficiently. This will both enable us to maximize the likelihood of finding the user's desired query within a limited time budget, and minimize the amount of time the user spends idly waiting for the system to search for queries.

Contributions - We offer the following contributions, extending a preliminary version of this work [3]:

(1) We propose the dual-specification query synthesis interaction model and introduce the table sketch query (TSQ) to enable users with domain knowledge to construct expressive SQL queries more accurately and efficiently than with previous single-specification approaches.

(2) We efficiently explore the search space of candidate queries with guided partial query enumeration (GPQE), which leverages a neural guidance model to enumerate the query search space and ascending-cost cascading verification in order to efficiently prune the search space. We describe our implementation of DuoQuest, a novel prototype dual-specification system, which leverages GPQE and a front-end web interface with autocomplete functionality for literal values.

(3) We present user studies on Duoouest demonstrating that the dual-specification approach enables a $62.5 \%$ absolute increase in accuracy over a state-of-the-art NLI and comparable accuracy to a PBE system on a more limited workload for the PBE system. We also present a simulation study on the Spider benchmark demonstrating a $>2 x$ increase in the top-1 accuracy of Duoguest over both NLI and PBE.

Organization - In Section 2, we provide an overview of our problem. We then describe our solution approach (Section 3) and system implementation (Section 4). We present our experimental evaluation, including user studies and simulated experiments (Section 5), explore related work (Section 6), discuss limitations of our approach and opportunities for future work (Section 7), and conclude (Section 8).

\section{PROBLEM OVERVIEW}

\subsection{Motivating Example}

Consider the following motivating example:

Example 2.1. Kevin wants to query a relational database containing movie information but has little knowledge of SQL or the schema. He issues the following NLQ to a NLI.

$N L Q$ : Show names of movies starring actors from before 1995, and those after 2000, with corresponding actor names, and years, from earliest to most recent.

\section{Sample Candidate SQL Queries:}

CQ1: Meaning: The names and years of movies released before 1995 or after 2000 starring male actors, with corresponding actor names, ordered from oldest to newest movie.

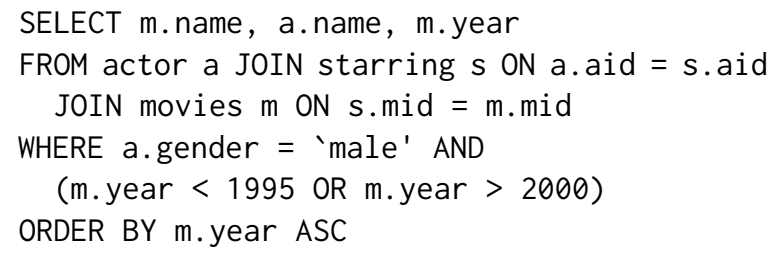

CQ2: Meaning: The names of movies starring actors/actresses born before 1995 or after 2000 and corresponding actor 
names and birth years, ordered from oldest to youngest actor/actress.

SELECT m.name, a.name, a.birth_yr

FROM actor a JOIN starring $s$ ON a.aid = s.aid

JOIN movies $\mathrm{m}$ ON s.mid $=\mathrm{m} . \mathrm{mid}$

WHERE a.birth_yr < 1995 OR a.birth_yr $>2000$

ORDER BY a.birth_yr ASC

CQ3: Meaning: The names and years of movies either (a) released before 1995 and starring male actors, or (b) released after 2000; with corresponding actor names, from oldest to newest movie.

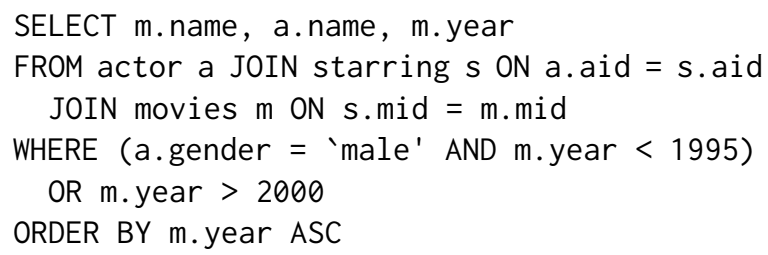

The NLI returns over 30 candidate queries. CQ3 is his desired query, but it is the 15 th ranked query returned by the NLI and not immediately visible in the interface.

Even for a human SQL expert, the NLQ in Example 2.1 is challenging to decipher, as each of the interpretations cannot be ruled out definitively without an explicit means of clarification by the user. In many cases, NLIs may not return the desired query in the top- $k$ displayed results, and users have no recourse other than to attempt to rephrase the NLQ without additional guidance from the system. In addition, leveraging a previous PBE system for Example 2.1 would be difficult unless Kevin already has a large number of exact, complete example tuples on hand.

With access to DuOQUEST, our dual-specification interface, Kevin can supply an optional PBE-like specification called a table sketch query (TSQ) to clarify his query, even with limited example knowledge:

Example 2.2. Kevin chooses to refine his natural language query with a table sketch query (TSQ) on DuoQuest.

He thinks of movies he knows well, and recalls that Tom Hanks starred in Forrest Gump before 1995 and that Sandra Bullock starred in Gravity sometime between 2010 and 2017. He encodes this information in the TSQ shown in Table 2.

Using the NLQ along with the TSQ, the system can eliminate CQ1 because it does not produce the second tuple (with Sandra Bullock, a female, starring in the movie), as well as CQ2, because Sandra Bullock was not born between 2010 and 2017. CQ3 is therefore correctly returned to Kevin.

The TSQ requires no schema knowledge from the user, allows users to specify partial tuples, and permits an openworld setting. When used alone, the TSQ is still likely to face the problem of an intractably large search space. However, when used together with an NLQ, the information from

\begin{tabular}{rccc}
\hline Types & text & text & number \\
\hline Tuples & & & \\
1. & Forrest Gump & Tom Hanks & \\
2. & Gravity & Sandra Bullock & [2010,2017] \\
\hline Sorted? & $\boldsymbol{x}$ & & \\
Limit? & None & & \\
\hline
\end{tabular}

Table 2: Example table sketch query (TSQ). Top: contains the data types for each column; Middle: example tuples; Bottom: indicates that desired query output will neither be sorted nor limited to top- $k$ tuples.

the natural language can guide the process to enable the synthesis of more expressive queries such as those including grouping and aggregates.

While the TSQ is optional, a dual-specification input is also preferred over the NLQ alone because it enables pruning of the search space of partial queries and permits a soundness guarantee that all returned results must satisfy the TSQ. In addition, the TSQ enables users a reliable, alternative means to refine queries iteratively (by adding additional tuples and other information to the TSQ) if their initial NLQ fails to return their desired query.

\subsection{Table Sketch Query}

We formally define the table sketch query (TSQ), which enables users to specify constraints on their desired SQL query at varied levels of knowledge in a similar fashion to existing PBE approaches [13, 14]. Unlike existing approaches, we also allow the user to include some additional metadata about their desired SQL query:

Definition 2.3. A table sketch query $\mathcal{T}=(\alpha, \chi, \tau, k)$ has:

(1) an optional list of type annotations $\alpha=\left(\alpha_{1}, \ldots, \alpha_{n}\right)$;

(2) an optional list of example tuples $\chi=\left(\chi_{1}, \ldots, \chi_{n}\right)$;

(3) a boolean sorting flag $\tau \in\{\top, \perp\}$ indicating whether the query should have ordered results; and

(4) an limit integer $k \geq 0$ indicating whether the query should be limited to the top- $k$ rows ${ }^{4}$.

A tuple in the result set of a query, $\chi_{q} \in R(q)$, satisfies an example tuple $\chi_{i}$ if each cell $\chi_{q}[j] \in \chi_{q}$ matches the corresponding cell of the same index $\chi_{i}[j] \in \chi_{i}$. As shown in Example 2.2, each example tuple $\chi_{i} \in \chi$ may contain exact cells, which match cells in $\chi_{q}$ of the same value; empty cells, which match cells in $\chi_{q}$ of any value, and range cells, which match cells in $\chi_{q}$ that have values within the specified range.

Definition 2.4. A query $q$ satisfies a TSQ $\mathcal{T}=(\alpha, \chi, \tau, k)$ if all of the following conditions are met:

(1) if $\alpha \neq \varnothing$, the projected columns of $q$ must have data types matching the annotations;

${ }^{4} k=0$ indicates no limit. 


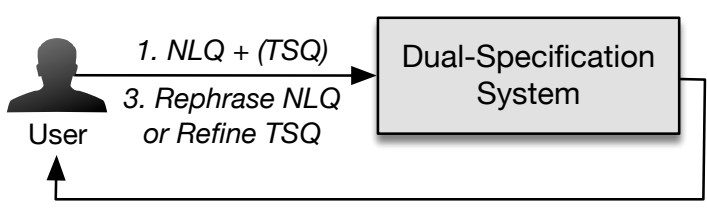

2. Candidate Queries

Figure 1: Dual-specification interaction model.

(2) if $\chi \neq \varnothing$, for each example tuple in $\chi$, there exists a distinct tuple in the result set of $q$ that satisfies it;

(3) if $\tau=\mathrm{T}, q$ must include a sorting operator and produce the satisfying tuples in (2) in the same order as the example tuples in the TSQ;

(4) if $k>0, q$ must return at most $k$ tuples.

We denote a table sketch query $\mathcal{T}(q, D)$ as a function taking a query $q$ and database $D$ as input. This function returns T if executing $q$ on $D$ satisfies $\mathcal{T}$, and $\perp$ otherwise.

\subsection{Problem Definition}

We now formally define our dual-specification problem:

Problem. Find the desired query $\hat{q}$ on database D, given:

(1) a natural language query $N$ describing $\hat{q}$, which includes a set of text and numeric literal values $L$ used in $\hat{q}$;

(2) an optional table sketch query $\mathcal{T}$ such that $\mathcal{T}(\hat{q}, D)=T$.

The literal values $L$ are a subset of tokens in the natural language query $N$. These can be obtained from the user by presenting an autocomplete-based tagging interface, as described further in Section 4.

\subsection{Interaction}

Figure 1 depicts the interaction model. The user issues a NLQ to the system, along with an optional TSQ. The system returns a ranked list of candidate queries. If none of candidate queries is the user's desired query, the user has two options: they may either rephrase their NLQ or refine their query by adding more information to the TSQ. This process continues iteratively until the user obtains their desired query.

\subsection{Task Scope}

We consider select-project-join-aggregate (SPJA) queries, including grouping, sorting, and limit operators. In clauses with multiple selection predicates, we disallow nested expressions with different logical operators such as a $>1$ OR (b $<1$ AND $c=1$ ) due to the challenge of expressing such predicates in a NLQ. For simplicity, we restrict join operations to inner joins on foreign key-primary key relationships, although alternate joins such as left joins can also be considered with minimal engineering effort.

\section{SOLUTION APPROACH}

\subsection{Overview}

The search space of possible SQL queries in our setting is enormous $^{5}$, with a long chain of inference decisions to be made about the presence of clauses, number of database elements in each clause, constants in expressions, join paths, etc. Discovering whether a single satisfying query exists for a set of examples, even in the context of select-projectjoin queries, is NP-hard [21]. The set of queries we hope to support only further expands this search space.

Previous work [20] attempts to tackle this challenge by implementing beam search, which limits the set of possible generated candidate queries to the $k$ highest-confidence branches at each inference step. However, this approach sacrifices completeness and can cause the correct query to be eliminated in cases where the model performs poorly.

By including the TSQ as an additional specification, we have an alternative means to prune the search space without sacrificing completeness. Consequently, we propose guided partial query enumeration (GPQE), which has two major features. First, GPQE performs guided enumeration by using the NLQ to guide the candidate SQL enumeration process, where candidates more semantically relevant to the NLQ are enumerated first. Second, GPQE leverages partial queries (PQs) as opposed to complete SQL queries to facilitate efficient pruning, defined as follows:

Definition 3.1. A partial query (PQ) is a SQL query in which a query element (i.e. SQL query, clause, expression, column reference, aggregate function, column reference, or constant) may be replaced by a placeholder.

Many NLI systems already generate PQs during query inference [22] or can be easily adapted [20] to do so. These PQs are tested against the TSQ to prune large branches of invalid queries early without needing to enumerate all complete queries in each branch, which is costly both because of the volume of complete queries and the time needed to verify each one. Ultimately, this enables the approach to cover more of the search space in a given amount of time.

\subsection{Algorithm}

Algorithm 1 describes the GPQE process, which takes in the natural language query $N$, an enumeration guidance model $M$, the table sketch query $\mathcal{T}$, and the database $D . P$ stores the collection of states to explore, where each state is a pair comprised of a partial query and a confidence score for that partial query (Line 2). On each iteration, $p$, the highest confidence state from $P$ is removed (Line 4). ENUMNEXTSTEP produces $Q$, the set of new partial query/confidence score

$\overline{{ }^{5} O\left(c^{n}\right) \text {, where }} c \geq 2$ is a constant determined by permitted expressivity and $n$ is the number of columns in the schema. 

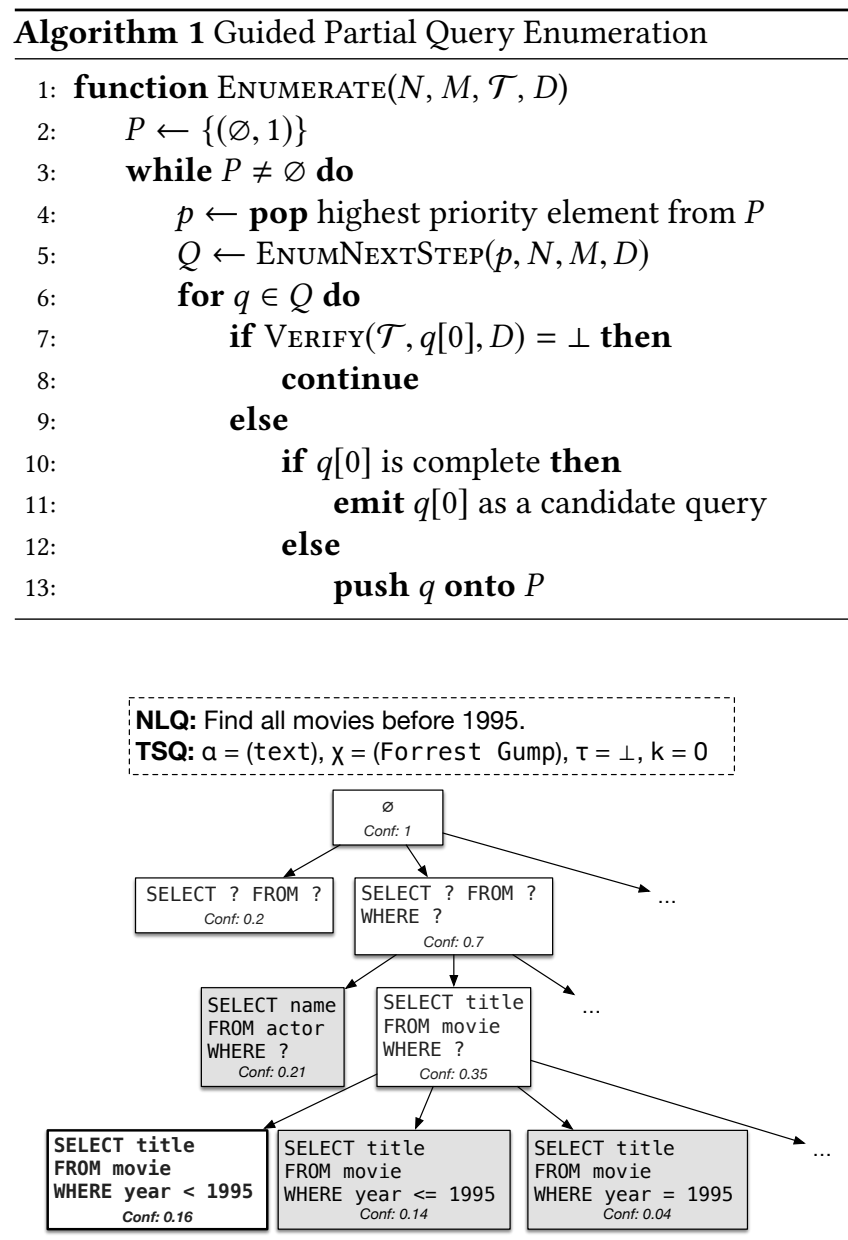

Figure 2: Simplified GPQE example. Each box is a state. Shaded boxes fail verification against the TSQ. The bolded state is the highest-ranked candidate query.

states that can be generated by making an incremental update to a single placeholder on the partial query in $p$ (Line 5). Each state $q \in Q$ is then verified against the table sketch query $\mathcal{T}$ (Line 7 ), and those that fail verification are discarded. The remaining states are examined to see whether they are complete queries (Line 10), in which case they are emitted as a valid candidate query (Line 11). Otherwise, they are pushed back onto $P$ for another iteration (Line 13). The candidate queries are returned to the user as a ranked list ordered from highest to lowest confidence score.

Figure 2 displays an example GPQE execution, where each box represents a state. Each new layer is an iteration, where candidate states are generated by ENUMNEXTSTEP using the highest-confidence state available at that iteration. Shaded boxes indicate that the state failed VERIFY. The highest-ranked candidate query is bolded.

\begin{tabular}{lll}
\hline Module & Responsibility & Output \\
\hline KW & $\begin{array}{l}\text { Clauses present in query (WHERE, } \\
\text { GROUP BY, ORDER BY) }\end{array}$ & Set \\
COL & Schema columns & Set \\
OP & Predicate operators (e.g. =, LIKE) & Set \\
AGG & Aggregate functions (MAX, MIN, & Set \\
& SUM, COUNT, AVG, None) & \\
AND/OR & Logical operators for predicates & Single \\
DESC/ASC & ORDER BY direction and LIMIT & Single \\
HAVING & Presence of HAVING clause & Single \\
\hline
\end{tabular}

Table 3: Selected modules from SyntaxSQLNet [23], their respective responsibility and output cardinality.

\subsection{Guided Enumeration}

In this section, we describe the enumeration process in ENUMNextStep. We adopt the SyntaxSQLNet [23] system and make several modifications to enable our approach to: (1) perform a complete enumeration over the possible search space, (2) perform a best-first search and robustly compare any two search states during enumeration, (3) perform verification of partial queries by fleshing out their join paths.

We begin by providing some necessary background knowledge of the SyntaxSQLNet system.

3.3.1 Background. SyntaxSQLNet uses a collection of recursive neural network modules, each responsible for making an enumeration decision for a specific SQL syntax element. We list the modules used in our system in Table 3. Each module takes the natural language query $N$, the partial query synthesized so far $p$, and optionally, the database schema $D$ (for modules such as the COL module which infer a column from the database schema). Given the input, each module returns the highest-confidence output class. For modules returning a set as output, a three-step decision is made: (1) a classifier predicts the number of values $k$ to return, (2) another classifier ranks the relevant output classes, and (3) the top- $k$ ranked classes are returned by the module.

The order of module execution is pre-assigned based on SQL syntax rules and the current output state $p$. For example, if a WHERE clause is being predicted, the COL, OP, and ROOT/TERM modules will be executed in order.

3.3.2 Candidate Enumeration. SyntaxSQLNet, by design, produces a single output query as output. To enable the search space enumeration in ENUMNEXTSTEP to be complete, we modify the modules in SyntaxSQLNet to produce all possible candidate states. We accomplish this by generating a new state for each candidate during each inference decision. For example, when executing the AND/OR module, we generate two candidate states, one each for AND and OR. For modules 


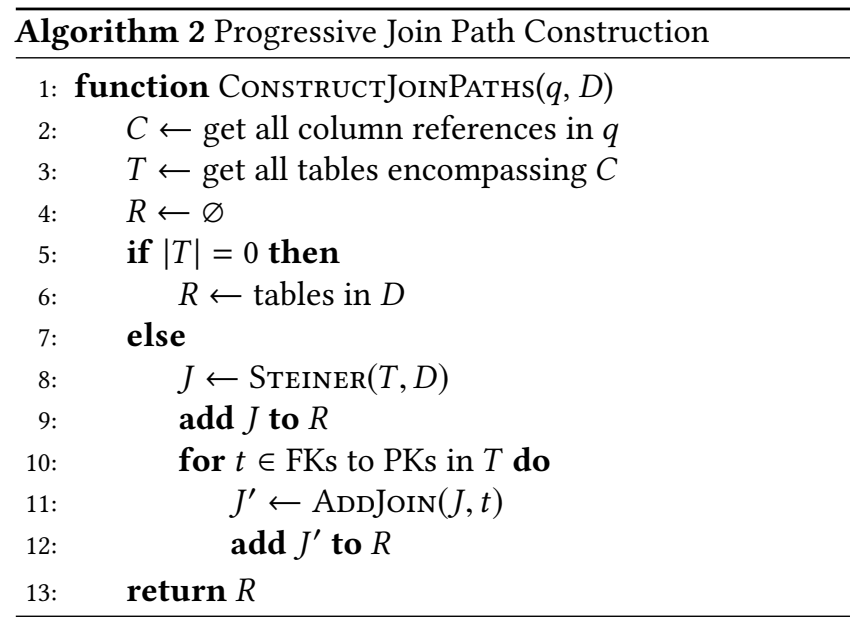

returning a set as output, the set of returned candidate states is the power set of the output classes.

3.3.3 Confidence Scores. SyntaxSQLNet produces rankings for each state with respect to its siblings in the search space by using the softmax function to produce a score in $(0,1)$ for each output class. However, to facilitate the best-first search in Line 4 of Algorithm 1, we need a overall confidence score that enables us to compare two states even if they are not siblings. As a result, we explicitly define the confidence score $C$ for a partial query state $p$ as follows:

$$
C(p)=\prod_{i=1}^{|p|} M\left(N, p_{i}, D\right)
$$

where each $p_{i}$ is the output class of the $i$-th inference decision made to generate the partial query in state $p$, and $M\left(N, p_{i}, D\right)$ is the softmax value returned by the appropriate SyntaxSQLNet module for NLQ $N$, output class $p_{i}$, on the schema of database $D$. In other words, the confidence score is the cumulative product of the softmax values of each output class comprising the partial query. Defining the confidence score in this way guarantees the following property:

Property 1. The sum of the confidence scores of all child branches of state $p$ is equal to the confidence score of $p$.

In theory, this confidence score definition also causes the system to prefer shorter queries over longer ones. Such concerns motivate previous systems [22] to adopt a confidence score definition motivated by the geometric mean. In practice, however, we found that this property of our confidence score did not negatively affect our system's ability to accurately synthesize user queries.

3.3.4 Progressive Join Path Construction. SyntaxSQLNet includes a rudimentary join path inference module to determine the tables and join conditions used in the FROM clause of a query. In SyntaxSQLNet, this join path module is (1) only applied to completed queries as the final step in the query inference process, and (2) only produces a single join path.

For our GPQE algorithm, however, we need join paths to be produced for each partial query, because the VERIFY procedure needs to be able to execute partial queries to compare them against the example tuples in the TSQ. In addition, user-provided NLQs often lack explicit information to guide the system to select one particular join path over another [2]. For this reason, and also to enable completeness in our search procedure, we produce all candidate join paths for each partial query rather than just a single join path.

To accomplish these goals, we adopt a technique called progressive join path construction. Algorithm 2 describes the join path construction process, which takes $q$, a partial query, and $D$, the database as input. First, the set of distinct tables encompassing all column references in $q$ are collected into $T$ (Line 3). If there are no tables present in the query (e.g. SELECT COUNT $(*))$, then each table in $D$ is returned as a candidate join path (Line 6). Otherwise, following the approach in [2], a Steiner tree is computed on the graph where nodes are tables and edges are foreign key to primary key relationships between the tables (Line 8). By default, all edge weights are set to 1 , though weights could also be derived from sources such as a query log [2]. Finally, in Lines 10-12, we add joins to cover cases where the desired query contains additional tables in the FROM clause beyond the columns already present in $q$, such as in the following example.

Example 3.2. A query utilizing more tables than those referenced outside the FROM clause:

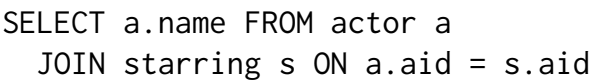

The process in Lines 10-12 can be recursively called to add joins of arbitrary depth. For simplicity, we only depict the process for one level of depth in Algorithm 2.

Whenever a new partial query is generated, progressive join path construction is executed to produce a new state for each candidate join path of the partial query. While all states produced by this process have the same confidence score, the enumeration process prioritizes states with higher confidence scores first, and then uses the join path length as a secondary tiebreaker, where shorter join paths are preferred.

3.3.5 Extensibility. As NLI models are undergoing rapid active development in the programming languages [22], natural language processing $[4,7,23]$, and database research communities [8], our approach is modular, enabling SyntaxSQLNet to be replaced by any NLI model that:

(1) is able to generate and incrementally apply updates to executable partial queries,

(2) emits a confidence score for each partial query in the range $[0,1]$ and fulfilling Property 1. 


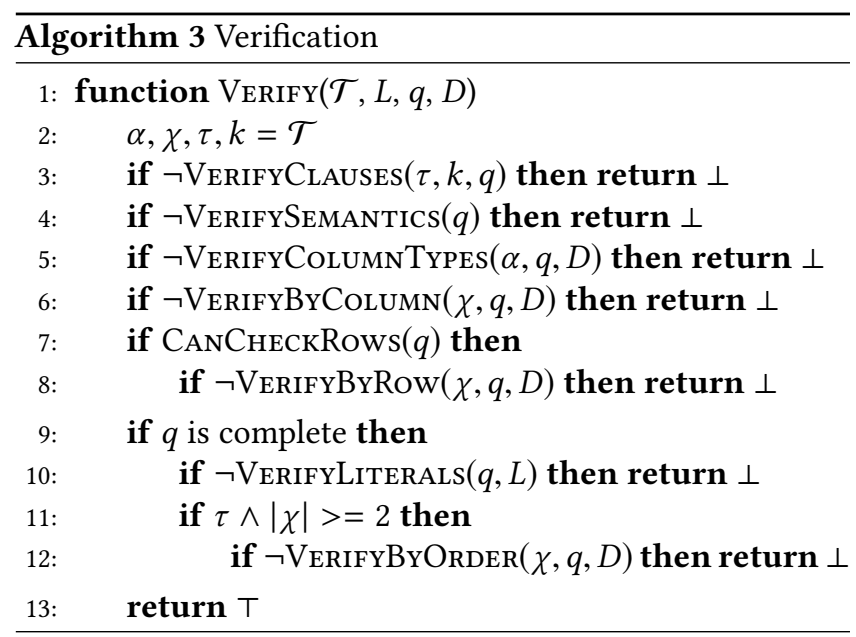

3.3.6 Scope. While SyntaxSQLNet supports set operations (INTERSECT, UNION, EXCEPT) and nested subqueries in predicates, we disabled this functionality to restrict output to the tasks described in Section 2.5.

\subsection{Verification}

During the enumeration process, verifying queries against the TSQ can be expensive for two reasons: (1) waiting until candidate queries are completely synthesized before verification causes redundant work to be performed on similar candidate queries, and (2) executing a single, complete candidate query on the database can be costly depending on the nature of the query and the database contents.

To mitigate these inefficiencies, we leverage ascending-cost cascading verification for the VERIFY function in Algorithm 1. Low-cost verifications, which do not require any access to the database $D$, are performed first to avoid performing highcost verifications, which involve issuing queries on $D$, until absolutely necessary. In addition, these verifications are performed as early as possible on partial queries in order to avoid performing redundant work on similar candidate queries. Algorithm 3 describes this process, which takes the TSQ $\mathcal{T}$, a partial query $q$, the literal values $L$ within the natural language query, and the database $D$ as input.

First, the presence of clauses is verified in VERIFYCLAUSES. If the TSQ specifies that results should be sorted or limited and the partial query does not match the TSQ, verification will fail. For example:

Example 3.3. Given a TSQ with sorting flag $\tau=\perp$ and the following partial queries, where ? indicates a placeholder:

CQ1: SELECT name, birth_yr FROM actor WHERE ?

CQ2: SELECT name, birthplace FROM actor WHERE ?

CQ3: SELECT a.name, COUNT(*) FROM actor a JOIN

starring $S$ ON a.aid = s. aid GROUP BY a.name

CQ4: SELECT a.name, MAX(m. revenue) FROM actor a
JOIN starring $s$ ON a.aid = s.aid JOIN

movies $\mathrm{m}$ ON m.mid = s.mid GROUP BY a. name

CQ5: SELECT name, debut_yr FROM actor ORDER BY ?

CQ5 would fail VERIFYCLAUSEs because the TSQ specifies that results are not to be ordered in the desired query, yet it contains an ORDER BY clause.

Second, semantic checks are performed on the query in VerifySemantics. This step constrains the search space by eliminating nonsensical or redundant yet syntacticallycorrect SQL queries. Over 40 such errors are cataloged in [5]. We check for a subset of these errors and some additional ones, listed in Table 4. While expert users may opt to intentionally write SQL queries that break some of these rules, we enforce these rules to constrain the set of produced queries to those even non-technical users can readily understand.

Third, the column types in the SELECT clause are verified against the types in the TSQ in VERIFYCOLUMNTYPES, which requires a check on the schema of $D$, but still without any need to query $D$ :

Example 3.4. Of the remaining queries CQ1-CQ4 in Example 3.3, given a TSQ with type annotations $\alpha=$ [text, number], CQ2 would fail VERIFyCOLUMNTyPes because the types of its projected columns in the SELECT clause are [text, text].

Fourth, in VerifyByColumn, tuples in the TSQ are compared column-wise against the SELECT clause of each partial query. This requires running relatively inexpensive columnwise verification queries on the database $D$ :

Example 3.5. Given an example tuple in the TSQ $\chi_{1}=$ [Tom Hanks, [1950, 1960]] and the queries CQ1, CQ3, and CQ4 from Example 3.3, VERIFyByColumn executes the following column-wise verification queries on the database:

CV1: SELECT 1 FROM actor WHERE name $=$ 'Tom Hanks' LIMIT 1

(for 1st projected column of CQ1, CQ3, and CQ4)

CV2: SELECT 1 FROM actor WHERE birth_yr >= 1950 AND birth_yr $<=1960$ LIMIT 1

(for 2nd projected column of CQ1)

CV3: SELECT 1 FROM movies WHERE revenue $>=1950$ AND revenue <= 1960 LIMIT 1

(for 2 nd projected column of CQ4)

$\mathrm{CV} 3$ is the only one producing an empty result set on $D$, thus causing CQ4 to fail VERIFYByCoLUmN.

For column-wise verification queries, SELECT 1 and LIMIT 1 are used to minimize the execution time on typical SQL engines. Each unaggregated projected column in the SELECT clause of the partial query is matched against the corresponding cell in the example tuple, whether via an equality operator for single-valued cells in the tuple or $>=/<=$ operators for range cells, and placed in the WHERE clause, while the 


\begin{tabular}{|c|c|c|c|}
\hline Error & Description & Example & Possible Alternative \\
\hline \multirow[t]{2}{*}{ Inconsistent predicates } & Do not permit selection predicates & SELECT name FROM actor & SELECT name FROM actor \\
\hline & $\begin{array}{l}\text { on the same column that contradict } \\
\text { each other. }\end{array}$ & $\begin{array}{l}\text { WHERE name }=\text { 'Tom Hanks' AND } \\
\text { name = 'Brad Pitt' }\end{array}$ & $\begin{array}{l}\text { WHERE name }=\text { 'Tom Hanks' OR } \\
\text { name = 'Brad Pitt' }\end{array}$ \\
\hline Constant output column & $\begin{array}{l}\text { Do not permit columns with equal- } \\
\text { ity predicates to be projected. }\end{array}$ & $\begin{array}{l}\text { SELECT name, birth_yr FROM } \\
\text { actor WHERE birth_yr }=1950\end{array}$ & $\begin{array}{l}\text { SELECT name FROM actor } \\
\text { WHERE birth_yr }=1950\end{array}$ \\
\hline \multirow[t]{2}{*}{ Ungrouped aggregation } & An unaggregated projection and ag- & SELECT birth_yr, COUNT(*) & SELECT birth_yr, $\quad$ COUNT $(*)$ \\
\hline & $\begin{array}{l}\text { gregation cannot be used together } \\
\text { without GROUP BY. }\end{array}$ & FROM actor & $\begin{array}{l}\text { FROM actor GROUP } \\
\text { birth_yr }\end{array}$ \\
\hline $\begin{array}{l}\text { GROUP BY with singleton } \\
\text { groups }\end{array}$ & $\begin{array}{l}\text { If each group consists of a single } \\
\text { row (e.g. group contains primary } \\
\text { key), aggregation is unnecessary. }\end{array}$ & $\begin{array}{l}\text { SELECT aid, MAX(birth_yr) } \\
\text { FROM actor GROUP BY aid }\end{array}$ & $\begin{array}{l}\text { SELECT aid, birth_yr FROM } \\
\text { actor }\end{array}$ \\
\hline Unnecessary GROUP BY & $\begin{array}{l}\text { If there are no aggregates in the } \\
\text { SELECT, ORDER BY or HAVING } \\
\text { clauses, GROUP BY is unnecessary. }\end{array}$ & $\begin{array}{lll}\text { SELECT name } & \text { FROM actor } \\
\text { GROUP BY name } & & \end{array}$ & SELECT name FROM actor \\
\hline Aggregate type usage & $\begin{array}{l}\text { MIN/MAX/AVG/SUM may not be ap- } \\
\text { plied to text columns. }\end{array}$ & SELECT AVG(name) FROM actor & $\mathrm{N} / \mathrm{A}$ \\
\hline \multirow[t]{4}{*}{ Faulty type comparison } & $>,<,>=,<=$, BETWEEN may not be & SELECT name FROM actor & $\mathrm{N} / \mathrm{A}$ \\
\hline & applied to text columns. & WHERE name >= 'Tom Hanks' & \\
\hline & $\begin{array}{l}\text { LIKE may not be applied to numeric } \\
\text { columns. }\end{array}$ & $\begin{array}{lcc}\text { SELECT birth_yr } & \text { FROM } \\
\text { actor WHERE birth_yr } & \text { LIKE }\end{array}$ & $\mathrm{N} / \mathrm{A}$ \\
\hline & & '\%1956\%' & \\
\hline
\end{tabular}

Table 4: List of semantic pruning rules. Rules may be modified depending on the domain and use case.

FROM clause is assigned as the table of the projected column. Aggregated projections with MIN or MAX are treated the same as unaggregated projections, as both these functions will produce an exact value from the projected column. For AVG, the range (i.e. minimum value to maximum value) of the projected column is compared with the range cell, and verification fails if the two ranges do not intersect. Projections with COUNT and SUM aggregations are ignored because no conclusion can easily be drawn for partial queries.

Fifth, row-wise verification is performed. CANCHECKRows enforces the precondition for row-wise verification: any partial query with aggregated projections needs completed WHERE/GROUP BY clauses with no holes, because completing those holes could change the output of the aggregated projections in the final query. Row-wise verification queries are similar to column-wise verification queries, except that they require output values of each partial query to reside in the same tuple when matched with example tuples in the TSQ:

Example 3.6. Given the example tuple $\chi_{1}$ from Example 3.5 and the queries CQ1 and CQ3 from Example 3.3, VERIFYBYRow executes the following row-wise verification queries on the database for CQ1 and CQ3 respectively:

RV1: SELECT 1 FROM actor WHERE name = 'Tom Hanks' AND (birth_yr $>=1950$ AND birth_yr $<=1960$ ) LIMIT 1

RV2: SELECT 1 FROM actor a JOIN starring $S$ ON a. aid $=$ s.aid WHERE name = 'Tom Hanks'
GROUP BY a. name HAVING (COUNT $(*)>=1950$ AND $\operatorname{COUNT}(*)<=1960) \operatorname{LIMIT} 1$

RV1 produces a valid result on $D$, while RV2 does not. As a result, CQ1 is the only CQ that passes all verification tests.

Each projected column in the SELECT clause of the candidate query is matched against the corresponding cell in the example tuple and appended to either the WHERE (for unaggregated projections) or HAVING (for aggregated projections) of the column-wise verification query. All other elements from the original candidate query (such as FROM, GROUP BY clauses, or other selection predicates) are retained in the row-wise verification query.

Finally, when the query $q$ is complete, the algorithm verifies that all literals $L$ are used in $q$ via VerIfyLiterals. Then, if multiple example tuples exist in the TSQ and the sorting flag $\tau=\mathrm{T}$, VERIFyByORDER executes $q$ on $D$ and ensures that each of the example tuples in $\chi$ is fulfilled in the same order as they were specified in the TSQ.

\subsection{Alternative Approaches}

Two naïve approaches to designing a dual-specification system are (1) intersecting the output of an NLI and PBE system and (2) chaining two systems so the output of one becomes the input of the next. The intersection approach is inefficient because each system will have to redundantly examine the search space without communicating with the other system. 


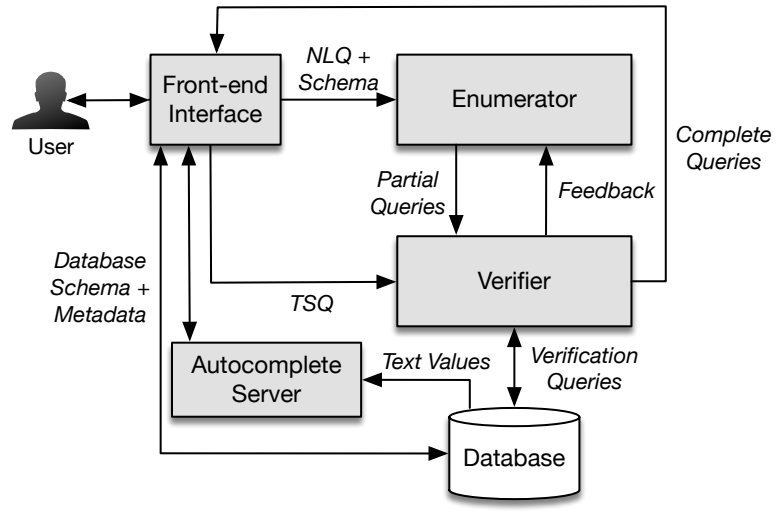

Figure 3: Architecture of Duoguest.

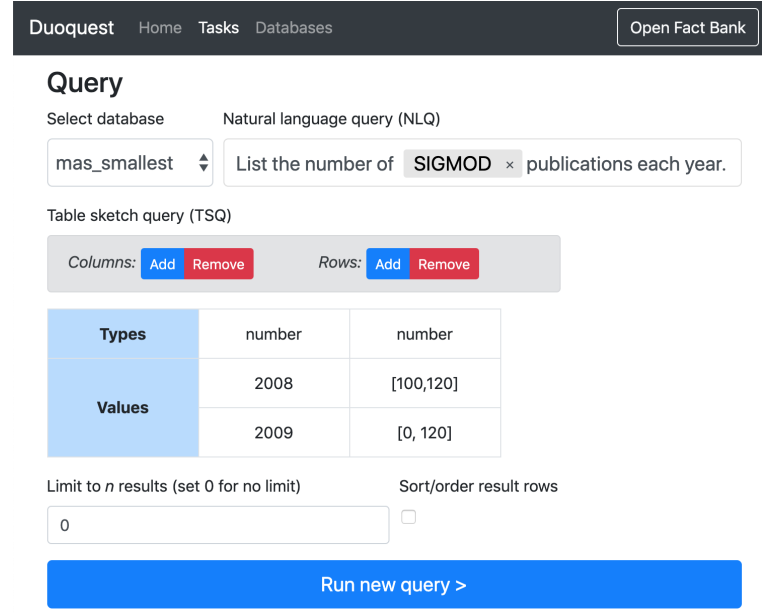

Figure 4: Screenshot of front-end interface. The "SIGMOD” tag was produced via autocomplete.

The chaining approach is more promising, where candidate queries generated by a NLI can be passed to a PBE system for verification, eliminating the redundancy in the intersection approach. However, it is still inefficient in comparison to GPQE, which enables us to eliminate large branches of complete queries by pruning partial queries.

\section{IMPLEMENTATION}

We implemented our approach in a prototype system, DuoQUEST $^{6}$. The system architecture (Figure 3 ) is comprised of 4 micro-services: the Enumerator, Verifier, Front-end Interface, and Autocomplete Server.

The Enumerator performs the ENUMNEXTSTEP procedure, and uses a SyntaxSQLNet [23] model pre-trained using the training and development sets of the cross-domain Spider dataset [24], while the Verifier service executes VERIFY.

\footnotetext{
$\overline{{ }^{6} \text { https://github.com/umich-dbgroup/duoquest }}$
}

The Front-End Interface (Figure 4) enables the user to specify queries. The interface contains a search bar for the user to specify the NLQ. Users can specify domain-specific literal text values in the NLQ search bar by typing the doublequote (") character, which activates an autocomplete search over a master inverted column index [16] containing all text columns in the database. The TSQ interface is below the search bar, where each cell in the interface activates the same autocomplete search as literal text values are typed.

After issuing the query, candidate SQL queries are displayed one at a time from highest to lowest confidence as the system enumerates and verifies them. Candidate queries continue to load until a pre-specified timeout is exceeded or the user clicks the "Stop Task" button. To enable users without knowledge of SQL to distinguish candidate queries and select from among them, each candidate query has a "Query Preview" button which executes the query on the database with LIMIT 20 appended to the query to retrieve a 20-row preview of the query results, and a "Full Query View" which executes the full query on the database.

\subsection{Domain-Specific Customization}

Adapting Duoquest to a new domain requires minimal effort, as the NLI model is trained on a cross-domain corpus. Additional domain-specific tasks can be used to retrain the model, and domain-specific semantic rules may also be appended to the default semantic rules provided by Duoguest. New databases should have foreign key-primary key constraints explicitly defined on the schema for the system to ingest (or these can be manually specified on our administrator's interface), and table and column names should use complete words rather than abbreviations (e.g. author_id instead of aid) as the NLI model relies on off-the-shelf word embedding models to interpret NLQs.

\section{EVALUATION}

We explored several research questions in our evaluation:

RQ1: Does the dual-specification approach help users to correctly synthesize their desired SQL query compared to single-specification approaches?

RQ2: Does the dual-specification approach conserve user effort over single-specification approaches?

RQ3: How does each component of our algorithm contribute to system performance?

RQ4: How does the amount of detail provided in the TSQ affect system performance?

\subsection{Setup for User Studies}

5.1.1 Compared Systems. For RQ1/RQ2, we conducted two within-subject user studies: one between Duoguest and 


\begin{tabular}{|c|c|c|c|c|c|c|c|c|c|}
\hline \multirow[b]{2}{*}{ Experiment } & \multirow[b]{2}{*}{ Dataset } & \multirow[b]{2}{*}{ Databases } & \multicolumn{4}{|c|}{ Tasks } & \multicolumn{3}{|c|}{ Avg. Schema Statistics } \\
\hline & & & Easy & Med & Hard & Total & Tables & Columns & FK-PK \\
\hline User Study vs. NLI & MAS [8] & 1 & 0 & 3 & 5 & 8 & 15 & 44 & 19 \\
\hline User Study vs. PBE & MAS [8] & 1 & 0 & 4 & 2 & 6 & 15 & 44 & 19 \\
\hline \multirow{2}{*}{ Simulation } & Spider Dev [24] & 20 & 239 & 252 & 98 & 589 & 4.1 & 22.1 & 3.2 \\
\hline & Spider Test [24] & 40 & 524 & 481 & 242 & 1247 & 4.5 & 19.6 & 3.6 \\
\hline
\end{tabular}

Table 5: Datasets used in our experiments, with the number of distinct databases and tasks per dataset, and the average number of tables, columns, and foreign key-primary key (FK-PK) relationships in all schemas. Easy tasks were project-join queries including aggregates, sorting, and limit operators, Medium tasks also included selection predicates, and Hard tasks included grouping operators.

SyntaxSQLNet [23], a state-of-the-art NLI; and the other with Duoquest and SQuID [6], a state-of-the-art PBE system.

We selected SyntaxSQLNet as a representative end-to-end neural network NLI. While some recent NLIs [4, 7] are known to outperform SyntaxSQLNet, their code was not available at the time of our study. In addition, their contributions are orthogonal to ours and can provide corresponding improvements to the guided enumeration process in DUOQUEST.

We selected SQuID as the representative PBE system because, to the best of our knowledge (Table 1), it is the only prominent PBE system that makes an open-world assumption, does not require schema knowledge of the user, and permits query expressivity beyond projections and joins.

For convenience, we denote SyntaxSQLNet as NLI and SQuID as $P B E$ for the remainder of this section.

5.1.2 Users. To reflect our motivation of supporting users with no specific knowledge of the schema and potentially without SQL experience, we recruited 16 users with no prior knowledge of the schema for our studies. Six of the users had little to no experience with SQL, while the remaining 10 had at least some experience with SQL.

5.1.3 Tasks. We tested DuoQuest against NLI on a variety of tasks within the scope described in Section 2.5. Since PBE did not support projected numeric columns or aggregates, we generated a second task set with a more limited scope of tasks for our study comparing DuoQuest and PBE.

We tested each user on the Microsoft Academic Search (MAS) database ${ }^{7}$ (Table 5) to see if they could synthesize the desired SQL query matching the provided task description. Each task description was provided in Chinese ${ }^{8}$ This resulted in a total of 128 task trials for the NLI study (64 on each system), and 96 task trials (48 on each system) for

\footnotetext{
${ }^{7}$ We removed some rows and columns unused in our tasks from the original database to reduce the user study time.

${ }^{8}$ All recruited subjects were bilingual in Chinese and English. following the study procedure in [8] to force the user to articulate the NLQ in English using their own words.
}

the PBE study. Users were given a time limit of 5 minutes for each task trial, which, in practice, was ample time for virtually all users to either complete the trial or give up after losing patience. Each user was given the same 2 tutorial tasks related to the actual task workload to try on each system prior to performing the study to teach them how to use each system.

The tasks (Appendix A) were split into two sets per user study (A/B for the NLI study and C/D for PBE). Half of the users were each given the first set to perform on DuoQuEsT first, then the second set to perform on the baseline system, while the other half of the users first attempted the first set on the baseline system, then the second set on Duouuest. The tasks in each set were given in the same order for each system, along with the 2 initial tutorial tasks, so that if there were any learning effects, they would happen equally on both systems. This means that results are comparable across systems for a given task, but not necessarily between two tasks.

5.1.4 Query Selection. NLI and DuoQuEsT produced a list of candidate SQL queries ranked from highest to lowest confidence, where each candidate query appeared as soon as the system enumerated it. Users with at least some SQL experience attempted to directly read the SQL queries before selecting one, as they could often understand the semantics of candidate queries even with no prior knowledge of the schema. On the other hand, users with little to no knowledge of SQL selected queries using a combination of eyeballing the selection predicates in the SQL queries and observing the "Query Preview" (described in Section 4) to view a sample of the result set of each candidate query as a sanity check.

In contrast to the other systems, PBE offered an "explanation" interface where users could check/uncheck suggested "filters" (i.e. selection predicates) to modify the produced query, with no need to consider the underlying SQL.

As a result, in the NLI study, both systems equally suffered from the same risk of users failing to properly understand the 
candidate SQL queries displayed to them. In the PBE study, the explanation interface arguably offered a slight advantage to PBE over DuoQuEsT for users with little knowledge of SQL. However, the study results demonstrated that the current interface was sufficient even for users without SQL knowledge to select the correct query on Duoduest.

5.1.5 Fact Bank. We designed our studies to explore the usability of each system given a fixed level of pre-existing domain knowledge in an open-world setting-i.e. where users only know a proper subset of tuples that will be produced by their desired query. To emulate such domain knowledge, we provided each user with a fact bank of 10 facts per task which was presented in randomly shuffled order during each trial. We allowed them to use any subset of these facts, but we did not allow them to use any knowledge external to the fact bank. These facts could be used in two ways: first, as example tuple input for DuoQuest or PBE; and second, as a means to verify the results of candidate queries by observing whether the facts reside in the produced output preview.

Each fact was provided as a sentence rather than as a tuple to require the user to discern how to input the fact into each system. For example, "List authors and their number of publications," a fact would be written in the form "Author X wrote 50 to 100 publications," and the user would figure out how to input this as (X, $[50,100])$ into DuoQuest.

A caveat of the fact bank design is that it does not test what happens when users provide incorrect examples. This may present a risk of bias particularly in our study with NLI, while in the study with PBE, both systems equally benefit from the fact bank. In a real world setting, the challenge of incomplete user knowledge is somewhat mitigated in DuoQuest by the autocomplete interface and the ability to provide partial or range examples. However, we acknowledge that further study is required to better investigate the effects of noisy examples on our system.

5.1.6 Environment. For DuoQuest and NLI, a server was set up on a Ubuntu 16.04 machine with $162.10 \mathrm{GHz}$ Intel Xeon Gold 6130 CPUs and 4 NVIDIA GeForce GTX $1080 \mathrm{Ti}$ GPUs (only a single GPU was used for inference), running PyTorch 0.4.0 on CUDA 7.5. The front end was accessed with a MacBook Pro using Google Chrome. PBE was executed on a Java graphical user interface on a MacBook Pro.

\subsection{User Study vs. NLI}

Figure 5 displays the proportion of the time users successfully completed each task. With regard to RQ1, it is clear that Duoduest enables users to discover the correct query far more frequently than the baseline NLI system, as only 15 out of $64(23.4 \%)$ trials were successful with NLI while that

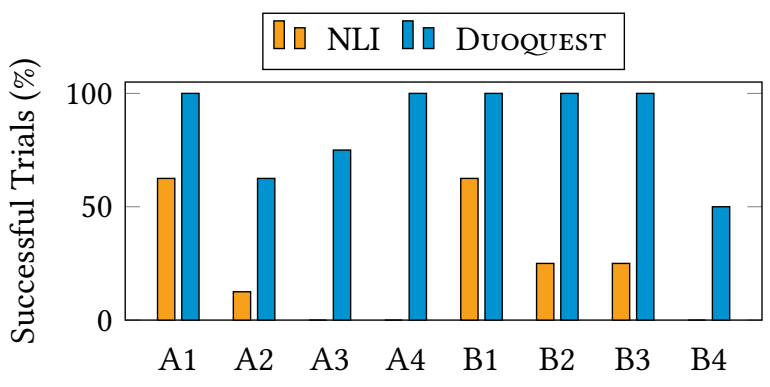

Figure 5: \% of trials for NLI study in which the user successfully completed each task within 5 minutes.

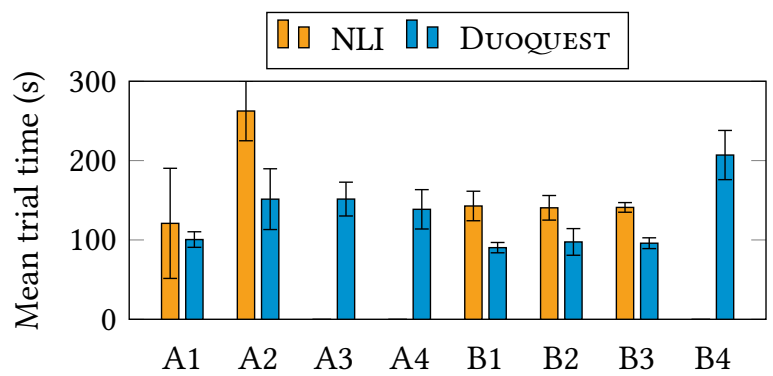

Figure 6: Mean time per task for correctly completed trials in NLI study, with error bars indicating standard error. A3, A4, B4 for NLI are omitted because there were no successful trials.

number shot up to 55 (85.9\%) for DuOQuEST, a $62.5 \%$ absolute increase in the percentage of task trials completed correctly. As evident from the figure, DuoQuest outperformed NLI on each individual task, with users failing to complete even a single trial on NLI for tasks A3, A4, B4. This is largely due to the additional PBE specification, which drastically shrinks the list of displayed candidate queries for Duoquest, while users grow fatigued manually verifying candidate queries in the large list for NLI.

For RQ2, we use user time as a metric for user effort, and observe in Figure 6 that DUOQUEST either reduces or requires comparable user effort to the baseline NLI system for every successful trial. This is also due to the reduction in the number of candidate queries displayed to the user.

Finally, the mean number of examples provided to DuoQUEST fell between 1 and 1.5 for each task, suggesting that DUOQUEST can be an effective tool for users even with just one or two examples regarding their desired query.

\subsection{User Study vs. PBE}

For RQ1, Figure 7 shows that DuOQUEST and PBE have comparable accuracy on the PBE-supported workload, 


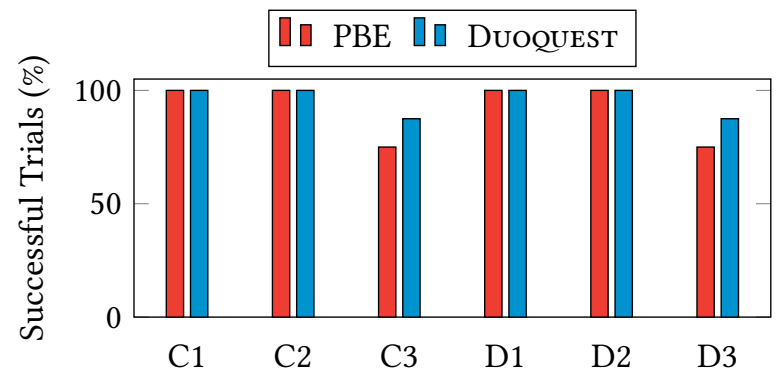

Figure 7:\% of trials for PBE study in which the user successfully completed each task within 5 minutes.

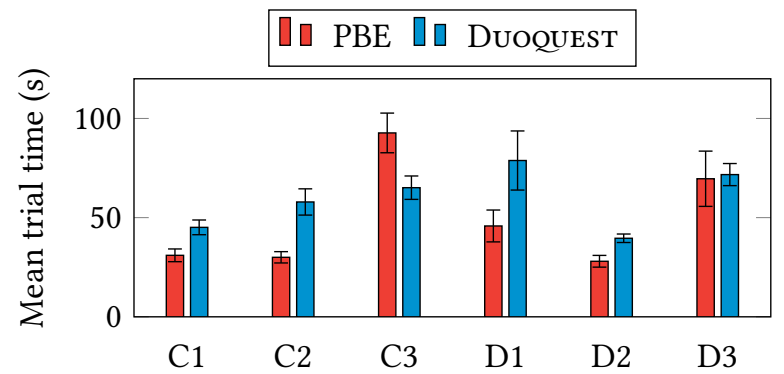

Figure 8: Mean time per task for correctly completed trials in PBE study; error bars for standard error.

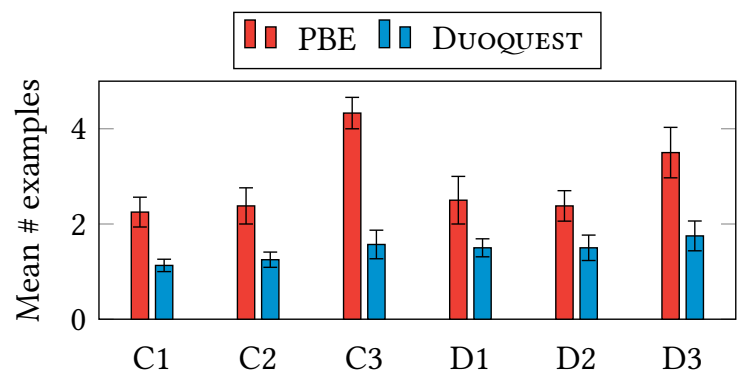

Figure 9: Mean \# examples used per task for successful trials in PBE study; error bars for standard error.

with Duoguest performing marginally better on the more difficult Hard tasks (C3, D3).

For RQ2, Figure 8 shows that user time is comparable for PBE and DUOQUEST on harder tasks but PBE is faster for simple tasks. $\mathrm{PBE}$ was faster for users on the easier Medium-level tasks (C1, C2, D1, D2) because of the time required for users to type out the NLQ on Duoguest. This additional cost was amortized for the more difficult Hard tasks (C3, D3) which contained aggregate operations due to the benefits gained by the additional NLQ specification.

Figure 9 displays how users issue more examples on average for PBE, suggesting that Duoguest may be preferred in cases when users know fewer examples if they are able to articulate an NLQ instead.

\begin{tabular}{|c|c|c|c|c|c|c|c|c|}
\hline \multirow[b]{2}{*}{ Sys. } & \multicolumn{2}{|c|}{ Top-1 } & \multicolumn{2}{|c|}{ Top-10 } & \multicolumn{2}{|c|}{ Correct } & \multicolumn{2}{|c|}{ Unsupp. } \\
\hline & \# & $\%$ & \# & $\%$ & $\#$ & $\%$ & \# & $\%$ \\
\hline DQ & 374 & 63.5 & 493 & 83.7 & - & - & 0 & 0 \\
\hline NLI & 178 & 30.2 & 334 & 56.7 & - & - & 0 & 0 \\
\hline PBE & - & - & - & - & 78 & 13.2 & 475 & 80.6 \\
\hline
\end{tabular}

(a) Spider Dev (589 total tasks)

\begin{tabular}{|c|c|c|c|c|c|c|c|c|}
\hline \multirow[b]{2}{*}{ Sys. } & \multicolumn{2}{|c|}{ Top-1 } & \multicolumn{2}{|c|}{ Top-10 } & \multicolumn{2}{|c|}{ Correct } & \multicolumn{2}{|c|}{ Unsupp. } \\
\hline & \# & $\%$ & \# & $\%$ & \# & $\%$ & \# & $\%$ \\
\hline DQ & 792 & 63.5 & 1065 & 85.4 & - & - & 0 & 0 \\
\hline NLI & 389 & 31.2 & 698 & 56.0 & - & - & 0 & 0 \\
\hline PBE & - & - & - & - & 203 & 16.3 & 972 & 77.9 \\
\hline
\end{tabular}

(b) Spider Test (1247 total tasks)

Figure 10: Top-1 and Top-10 accuracy for Duoeuest (DQ) and NLI, task correctness for PBE, and amount of unsupported tasks.

\subsection{Simulation Study}

5.4.1 Setup. We evaluated Duoquest on the Spider benchmark [24], which is comprised of 10,181 NLQ-SQL pairs on 200 databases split into training (7,000 tasks), development (1,034 tasks), and test (2,147 tasks) sets. We removed tasks for which the SQL produced an empty result set or was outside our task scope (Section 2.5), or if the database had annotation errors (e.g. incorrect data types or integrity constraints in the schema). The final development and test sets we tested on (Table 5) had 589 tasks and 1,247 tasks, respectively.

For each task, the SQL label from the Spider benchmark was designated as the user's desired query, and literal values used within the SQL label were set to be the input literals $L$. We synthesized TSQs for each task, where each of the TSQs contained type annotations, two example tuples randomly selected from the result set of the desired SQL query, and $\tau$ and $k$ values corresponding to the desired query.

We compared the 3 systems from the user studies: DuoQUEST; SyntaxSQLNet (NLI); and SQuID (PBE). For each task, Duoguest was given the NLQ, literals, and synthesized TSQ; NLI was given the NLQ and literals; and PBE was given the example tuples of the synthesized TSQ. The systems were run on the same machines as the user study.

DUOQUEST and NLI produced a ranked list of candidate queries one at a time from highest to lowest confidence. The task was terminated when the desired query was produced by the system or a timeout of 60 seconds was reached. On the other hand, PBE returned a single set of projected columns with multiple candidate selection predicates at a single point in time, with a mean runtime of 1.7 seconds for the development set and 0.7 seconds for the test set. 


\begin{tabular}{|c|c|c|c|c|c|c|c|c|c|}
\hline \multirow[b]{2}{*}{ Sys. } & \multicolumn{3}{|c|}{ Easy } & \multicolumn{3}{|c|}{ Medium } & \multicolumn{3}{|c|}{ Hard } \\
\hline & $\checkmark \#$ & $\sqrt{ } \%$ & U\# & $\checkmark \#$ & $\checkmark \%$ & U\# & $\checkmark \#$ & $\checkmark \%$ & U\# \\
\hline $\mathrm{DQ}_{\mathrm{Q}}$ & 218 & 91.2 & 0 & 214 & 84.9 & 0 & 61 & 62.2 & 0 \\
\hline NLI & 158 & 66.1 & 0 & 143 & 56.8 & 0 & 33 & 33.8 & 0 \\
\hline PBE & 29 & 12.1 & 210 & 49 & 19.4 & 167 & 0 & 0 & 98 \\
\hline
\end{tabular}

(a) Spider Dev (239 easy, 252 medium, 98 hard tasks)

\begin{tabular}{|c|c|c|c|c|c|c|c|c|c|}
\hline \multirow[b]{2}{*}{ Sys. } & \multicolumn{3}{|c|}{ Easy } & \multicolumn{3}{|c|}{ Medium } & \multicolumn{3}{|c|}{ Hard } \\
\hline & $\sqrt{ } \#$ & $\sqrt{ } \%$ & U\# & $\sqrt{ } \#$ & $\sqrt{2} \%$ & U\# & $\sqrt{ } \#$ & $\sqrt{ } \%$ & U\# \\
\hline DQ & 495 & 94.5 & 0 & 407 & 84.6 & 0 & 163 & 67.4 & 0 \\
\hline NLI & 379 & 72.3 & 0 & 246 & 51.1 & 0 & 73 & 30.2 & 0 \\
\hline PBE & 107 & 20.4 & 417 & 96 & 20.0 & 313 & 0 & 0 & 242 \\
\hline
\end{tabular}

(b) Spider Test (524 easy, 481 medium, 242 hard tasks)

Figure 11: Number $(\checkmark \#)$ and proportion $(\checkmark \%)$ of correct tasks (top-10 accuracy for $D Q$ and NLI) and number of unsupported tasks (U\#) by task difficulty level.

5.4.2 Accuracy. Figure 10 displays the results of DuoQuest and NLI's top- $k$ accuracy, which is the number of tasks for which the desired query appeared in the top- $k$ of returned candidate queries. In particular, the Top-10 accuracy is a good proxy for the user's ability to discover their desired query, as we consider that examining a list of 10 candidate queries is a reasonable burden for the user to carry.

The PBE system was unable to handle a large proportion of our benchmark tasks because it did not support projections of numeric columns or aggregate values and selection predicates with negation or LIKE operators. For tasks the PBE system could support, we did not measure top- $k$ accuracy because the expected interaction model differed from the other systems. Instead, we labeled the result Correct if the selection predicates in the desired query were a subset of PBE's produced candidate selection predicates, ignoring any differences in specific literal values.

Reinforcing our conclusions on RQ1 from the user study, DuoQUest handily beats single-specification approaches NLI and PBE, with $\boldsymbol{a}>\mathbf{2 x}$ increase in Top-1 accuracy and 47.6\% increase in Top-10 accuracy over NLI, and an even larger improvement over PBE on the development set. Results are similar on the test set.

Figure 11 presents a breakdown of task success by difficulty level, measured by top-10 accuracy for Duoguest and NLI and correctness for PBE. As expected, systems perform generally worse on more difficult tasks as the resulting SQL for harder tasks contained more complex query constructs. $\mathrm{PBE}$ was unable to support any hard tasks because they all included projected aggregate values.

While PBE should have been able to get all supported tasks correct, it failed several tasks to due to its requirements

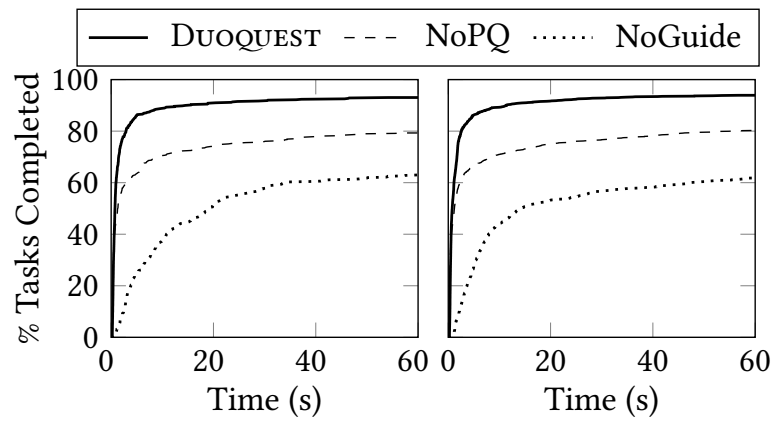

(a) Spider Dev

(b) Spider Test

Figure 12: Distributions of the time taken for each algorithm to synthesize the correct query. A higher curve indicates superior performance.

for a star/snowflake schema and user-defined metadata annotations as to which schema attributes are "entities" or "concepts". While we offered our best effort in restructuring and labeling the schema so as to support all given tasks, we found that for some schemas, all tasks for the schema could not be simultaneously supported with any schema structure given the current system design.

5.4.3 Guided Partial Query Enumeration (GPQE). To answer RQ3, we selectively disabled the two components of the GPQE algorithm used in Duoguest: guided enumeration (Section 3.3) and pruning of partial queries (Section 3.4). The version without guided enumeration (NoGuide) used only the literals from the NLQ specification and performed a naïve breadth-first search enumeration of all possible queries (ignoring confidence scores) while still pruning partial queries when possible. Simpler queries (i.e. those with less operations) were enumerated first and column attributes were enumerated following the order of the schema metadata provided in the Spider benchmark. The algorithm disabling pruning of partial queries $(N o P Q)$ leveraged enumeration guidance, but only verified complete queries, not partial ones, making it identical to the naïve chaining approach described in Section 3.5.

Figure 12 displays the results. In theory, all these systems explore the same search space, and given enough time, the distributions will all converge. In practice, however, the user cannot wait indefinitely, and the figure demonstrates how performance suffers immensely when we disable either guided enumeration or the pruning of partial queries, highlighting their necessity in facilitating an efficient, interactive-time system.

5.4.4 Specification Detail. To answer RQ4, we varied the amount of detail in the synthesized TSQ provided to DuoQUEST. We considered three different levels of detail: 


\begin{tabular}{rccccccc}
\hline & \multicolumn{3}{c}{ Spider Dev } & & \multicolumn{3}{c}{ Spider Test } \\
\cline { 2 - 3 } \cline { 7 - 8 } Detail & T1 & T10 & T100 & & T1 & T10 & T100 \\
\hline Full & $\mathbf{6 3 . 5}$ & $\mathbf{8 3 . 7}$ & $\mathbf{9 1 . 7}$ & & $\mathbf{6 3 . 5}$ & $\mathbf{8 5 . 4}$ & $\mathbf{9 2 . 4}$ \\
Partial & 59.6 & 77.1 & 90.3 & & 58.6 & 81.5 & 90.5 \\
Minimal & 40.8 & 60.6 & 85.9 & & 41.1 & 68.6 & 85.1 \\
NLI & 30.2 & 56.7 & 69.4 & & 31.2 & 56.0 & 69.5 \\
\hline
\end{tabular}

Table 6: Top-1, Top-10, and Top-100 exact matching accuracy (\%) for TSQs with varying amounts of specification detail. NLI results shown for comparison.

(1) Full, using the full synthesized TSQ described in Section 5.4.1;

(2) Partial, for which all values for a randomly-selected single column in tasks with at least 2 projected columns were erased from example tuples in the Full TSQ;

(3) Minimal, which removes all example tuples from the TSQ, leaving only column type annotations.

Table 6 demonstrates how an increase in specification detail helps contribute to a corresponding increase in the performance of Duoquest. Performance for the Partial TSQ has a relatively small dropoff from the Full TSQ, showing the promise of using partial or incomplete tuple knowledge to help users construct queries. There is a larger gap between Partial and Minimal TSQs, suggesting that the presence of even a single partial tuple is preferable to no example tuples at all. Finally, even providing type annotations for each column allows a $30 \%$ improvement in top-1 accuracy over the baseline NLI system which uses no TSQ.

\section{RELATED WORK}

Natural language interfaces. Most early natural language interfaces for relational databases were confined to a single domain [1]. Later work focused on the general-purpose case for easy adoption on arbitrary schemas. The Precise system explicitly defined "semantic coverage" to constrain the scope of natural language that could be expressed [12]. Other systems utilized different technologies such as dependency parse trees [8], semantic parsing [22], or pre-defined ontologies [15] to expand the scope of expressible queries. More recently, advances in deep learning have given rise to a new approach of building end-to-end deep learning systems to translate natural language queries to SQL. The current state-of-the-art utilizes techniques such as a modular syntax tree network [23], graph neural networks [4], or an intermediate representation [7] to generate SQL queries of arbitrary complexity. Our dual-specification approach alleviates ambiguity in natural language by allowing the user to provide a table sketch query to constrain the query search space.

Programming-by-example (PBE) systems. These interfaces permit users to provide a set of example output tuples or the full output of the desired query to search for queries on the database. A large body of work exists in this area [10], a representative sample of which is displayed in Table 1 . Such systems often have to sacrifice query complexity or enforce requirements on user knowledge (schema knowledge; full, exact tuples; or a closed-world setting) to make the search problem tractable. More recent work [6] has made an attempt to discern query intent in $\mathrm{PBE}$ with complex queries using pre-computed statistics and semantic properties. Our dual-specification approach tackles the same challenge in an orthogonal manner by leveraging the user's natural language query in addition to the user-provided examples.

\section{LIMITATIONS AND FUTURE WORK}

In this section, we identify some potential limitations and improvements to the current DuoQuEsT prototype.

First, additional work needs to be done to produce a completely SQL-less interaction model. Currently, users interact with produced candidate SQL queries to select their final query. During our evaluation, users without knowledge of SQL or the schema used various signals to assess whether a candidate query was the desired one (Section 5.1.4), and they were for the most part successful. Users' success may vary, however, when working with schemas with confusing attribute names or with highly complex SQL queries. As a result, there is a need for an interaction model that permits users to validate produced candidate SQL queries against their domain knowledge without exposing the actual SQL syntax to them.

Second, Duoquest is not yet able to deal with noisy (i.e. incorrect) examples. In the real world, users are often prone to errors and misinformation, and while this is mitigated somewhat by the autocomplete feature in Duoguest, techniques such as error detection or probabilistic reasoning should be implemented to enable Duoeuest to handle noisy examples.

Finally, Duoquest can be improved by streamlining iterative interaction. For example, the current interface could be improved by enabling users to add positive or negative examples to the TSQ specification by clicking a button directly on a candidate query preview. In addition, enabling users to directly modify generated candidate queries, perhaps by presenting them in some intermediate representation, would allow greater flexibility in synthesizing queries than merely having the user select from the system-generated list.

\section{CONCLUSION}

In this paper, we proposed dual-specification query synthesis, which consumes both a NLQ and an optional PBE-like table sketch query enabling users to express varied levels of knowledge. We introduced the guided partial query enumeration (GPQE) algorithm to synthesize queries from a 
dual-mode specification, and implemented GPQE in a novel prototype system Duoquest. We presented results from a user study in which Duoduest enabled a $62.5 \%$ absolute increase in query construction accuracy over a state-of-the-art $\mathrm{NLI}$ and comparable accuracy to a PBE system on a more limited workload supported by the PBE system. In a simulation study, Duoguest demonstrated a $>2 \mathrm{x}$ increase in top- 1 accuracy over both NLI and PBE.

\section{ACKNOWLEDGEMENTS}

We are grateful for a University of Michigan MIDAS grant to fund this work. We also thank Tao Yu, Bo Pang, and the Yale LILY Lab for assisting us with executing DUoQuest on the Spider dataset.

\section{REFERENCES}

[1] I. Androutsopoulos, G. D. Ritchie, and P. Thanisch. Natural language interfaces to databases - an introduction. Natural Language Engineering, 1(1):29-81, 1995.

[2] C. Baik, H. V. Jagadish, and Y. Li. Bridging the semantic gap with SQL query logs in natural language interfaces to databases. In 35th IEEE International Conference on Data Engineering, ICDE 2019, Macao, China, April 8-11, 2019, pages 374-385. IEEE, 2019.

[3] C. Baik, Z. Jin, M. J. Cafarella, and H. V. Jagadish. Constructing expressive relational queries with dual-specification synthesis. In CIDR 2020, 10th Conference on Innovative Data Systems Research, Amsterdam, The Netherlands, January 12-15, 2020, Online Proceedings, 2020.

[4] B. Bogin, J. Berant, and M. Gardner. Representing schema structure with graph neural networks for text-to-SQL parsing. In Proceedings of the 57th Conference of the Association for Computational Linguistics, ACL 2019, Florence, Italy, fuly 28-August 2, 2019, Volume 1: Long Papers, pages 4560-4565, 2019.

[5] S. Brass and C. Goldberg. Semantic errors in SQL queries: A quite complete list. Journal of Systems and Software, 79(5):630-644, 2006.

[6] A. Fariha and A. Meliou. Example-driven query intent discovery: Abductive reasoning using semantic similarity. PVLDB, 12(11):12621275, 2019.

[7] J. Guo, Z. Zhan, Y. Gao, Y. Xiao, J. Lou, T. Liu, and D. Zhang. Towards complex text-to-sql in cross-domain database with intermediate representation. In Proceedings of the 57th Conference of the Association for Computational Linguistics, ACL 2019, Florence, Italy, fuly 28-August 2, 2019, Volume 1: Long Papers, pages 4524-4535, 2019.

[8] F. Li and H. V. Jagadish. Constructing an interactive natural language interface for relational databases. PVLDB, 8(1):73-84, 2014.

[9] H. Li, C. Chan, and D. Maier. Query from examples: An iterative, data-driven approach to query construction. PVLDB, 8(13):2158-2169, 2015.

[10] D. M. L. Martins. Reverse engineering database queries from examples: State-of-the-art, challenges, and research opportunities. Inf. Syst., 83:89-100, 2019.

[11] K. Panev and S. Michel. Reverse engineering top-k database queries with PALEO. In Proceedings of the 19th International Conference on Extending Database Technology, EDBT 2016, Bordeaux, France, March 15-16, 2016, Bordeaux, France, March 15-16, 2016., pages 113-124, 2016.

[12] A. Popescu, O. Etzioni, and H. A. Kautz. Towards a theory of natural language interfaces to databases. In Proceedings of the 8th International Conference on Intelligent User Interfaces, IUI 2003, Miami, FL, USA, January 12-15, 2003, pages 149-157, 2003.
[13] F. Psallidas, B. Ding, K. Chakrabarti, and S. Chaudhuri. S4: top-k spreadsheet-style search for query discovery. In Proceedings of the 2015 ACM SIGMOD International Conference on Management of Data, Melbourne, Victoria, Australia, May 31 - June 4, 2015, pages 2001-2016, 2015.

[14] L. Qian, M. J. Cafarella, and H. V. Jagadish. Sample-driven schema mapping. In Proceedings of the ACM SIGMOD International Conference on Management of Data, SIGMOD 2012, Scottsdale, AZ, USA, May 20-24, 2012, pages 73-84, 2012.

[15] D. Saha, A. Floratou, K. Sankaranarayanan, U. F. Minhas, A. R. Mittal, and F. Özcan. ATHENA: an ontology-driven system for natural language querying over relational data stores. PVLDB, 9(12):1209-1220, 2016.

[16] Y. Shen, K. Chakrabarti, S. Chaudhuri, B. Ding, and L. Novik. Discovering queries based on example tuples. In International Conference on Management of Data, SIGMOD 2014, Snowbird, UT, USA, fune 22-27, 2014, pages 493-504, 2014.

[17] W. C. Tan, M. Zhang, H. Elmeleegy, and D. Srivastava. REGAL+: reverse engineering SPJA queries. PVLDB, 11(12):1982-1985, 2018.

[18] Q. T. Tran, C. Y. Chan, and S. Parthasarathy. Query reverse engineering. VLDB J., 23(5):721-746, 2014.

[19] C. Wang, A. Cheung, and R. Bodík. Synthesizing highly expressive SQL queries from input-output examples. In Proceedings of the 38th ACM SIGPLAN Conference on Programming Language Design and Implementation, PLDI 2017, Barcelona, Spain, June 18-23, 2017, pages 452-466, 2017.

[20] C. Wang, P. Huang, A. Polozov, M. Brockschmidt, and R. Singh. Execution-guided neural program decoding. CoRR, abs/1807.03100, 2018.

[21] Y. Y. Weiss and S. Cohen. Reverse engineering SPJ-queries from examples. In Proceedings of the 36th ACM SIGMOD-SIGACT-SIGAI Symposium on Principles of Database Systems, PODS 2017, Chicago, IL, USA, May 14-19, 2017, pages 151-166, 2017.

[22] N. Yaghmazadeh, Y. Wang, I. Dillig, and T. Dillig. SQLizer: query synthesis from natural language. PACMPL, 1(OOPSLA):63:1-63:26, 2017.

[23] T. Yu, M. Yasunaga, K. Yang, R. Zhang, D. Wang, Z. Li, and D. R. Radev. SyntaxSQLNet: syntax tree networks for complex and cross-domain text-to-SQL task. In Proceedings of the 2018 Conference on Empirical Methods in Natural Language Processing, Brussels, Belgium, October 31 - November 4, 2018, pages 1653-1663, 2018.

[24] T. Yu, R. Zhang, K. Yang, M. Yasunaga, D. Wang, Z. Li, J. Ma, I. Li, Q. Yao, S. Roman, Z. Zhang, and D. R. Radev. Spider: A large-scale humanlabeled dataset for complex and cross-domain semantic parsing and text-to-SQL task. In Proceedings of the 2018 Conference on Empirical Methods in Natural Language Processing, Brussels, Belgium, October 31 - November 4, 2018, pages 3911-3921, 2018.

[25] M. M. Zloof. Query by example. In American Federation of Information Processing Societies: 1975 National Computer Conference, 19-22 May 1975, Anaheim, CA, USA, pages 431-438, 1975.

\section{A USER STUDY TASKS}

Table 7 and Table 8 respectively contain the full list of tasks for the NLI and PBE user studies. 


\begin{tabular}{|c|c|c|c|}
\hline Task & Level & English Description & SQL \\
\hline A1 & M & $\begin{array}{l}\text { List all publications in conference } \\
\mathrm{C} \text { and their year of publication. }\end{array}$ & $\begin{array}{l}\text { SELECT t2.title, t2. year FROM conference AS t1 JOIN publication AS } \\
\text { t2 ON t1.cid = t2. cid WHERE t1. name = ' } \mathrm{C} \text { ' }\end{array}$ \\
\hline A2 & $\mathrm{H}$ & $\begin{array}{l}\text { List keywords and the number of } \\
\text { publications containing each, or- } \\
\text { dered from most to least publica- } \\
\text { tions. }\end{array}$ & $\begin{array}{l}\text { SELECT t1.keyword, COUNT }(*) \text { FROM keyword AS t1 JOIN } \\
\text { publication_keyword AS t2 ON t1.kid }=\text { t2. kid JOIN publication AS } \\
\text { t3 ON t2.pid = t3.pid GROUP BY t1.keyword ORDER BY count }(*) \text { DESC }\end{array}$ \\
\hline A3 & $\mathrm{H}$ & $\begin{array}{l}\text { How many publications has each } \\
\text { author from organization R pub- } \\
\text { lished? }\end{array}$ & $\begin{array}{l}\text { SELECT t1. name, COUNT }(*) \text { FROM author AS t1 JOIN writes AS t2 ON } \\
\text { t2.aid = t1.aid JOIN organization AS t3 ON t3.oid = t1. oid JOIN } \\
\text { publication t } 4 \text { ON t4.pid = t2.pid WHERE t3. name = ' } R \text { ' GROUP BY } \\
\text { t1. name }\end{array}$ \\
\hline A4 & $\mathrm{H}$ & $\begin{array}{l}\text { List journals with more than } 500 \\
\text { publications and the publication } \\
\text { count for each. }\end{array}$ & $\begin{array}{l}\text { SELECT DISTINCT t1. "name", COUNT }(*) \text { FROM journal AS t1 JOIN } \\
\text { publication AS t2 ON t1.jid }=\text { t2.jid GROUP BY t1. name HAVING } \\
\text { COUNT }(*)>500\end{array}$ \\
\hline B1 & $\mathrm{M}$ & $\begin{array}{l}\text { List the titles and years of publi- } \\
\text { cations by author A. }\end{array}$ & $\begin{array}{l}\text { SELECT t1.title, t1.year FROM publication AS t1 JOIN writes AS } \\
\text { t2 ON t2.pid = t1.pid JOIN author AS t3 ON t3.aid = t2. aid WHERE } \\
\text { t3. name = ' } A \text { ' }\end{array}$ \\
\hline $\mathrm{B} 2$ & M & $\begin{array}{l}\text { List the conferences and home- } \\
\text { pages in the D domain. }\end{array}$ & $\begin{array}{l}\text { SELECT t1. name, t1.homepage FROM conference AS t1 JOIN } \\
\text { domain_conference AS t2 ON t2.cid = t1.cid JOIN domain AS t3 oN } \\
\text { t3.did = t2.did WHERE } t 3 . \text { name }=\text { ' } D \text { ' }\end{array}$ \\
\hline B3 & $\mathrm{H}$ & $\begin{array}{l}\text { List organizations with more } \\
\text { than } 100 \text { authors and the number } \\
\text { of authors for each. }\end{array}$ & $\begin{array}{l}\text { SELECT t2. name, COUNT }(*) \text { FROM author AS t1 JOIN organization AS t2 } \\
\text { ON t1.oid }=\text { t2. oid GROUP BY t2. name HAVING COUNT }(*)>100\end{array}$ \\
\hline B4 & $\mathrm{H}$ & $\begin{array}{l}\text { List authors from organization } \mathrm{R} \\
\text { with more than } 50 \text { publications } \\
\text { and the number of publications } \\
\text { for each author. }\end{array}$ & $\begin{array}{l}\text { SELECT t1. name, COUNT }(*) \text { FROM author AS t1 JOIN writes AS t2 ON } \\
\text { t1. aid }=\text { t2.aid JOIN organization AS t3 ON t1.oid }=\text { t3. oid JOIN } \\
\text { publication AS t4 ON t2.pid = t4.pid WHERE t3. name }=\text { ' } \text { ' GROUP BY } \text { t1. name HAVING COUNT }(*)>50\end{array}$ \\
\hline
\end{tabular}

Table 7: Tasks for the user study vs. NLI, with abbreviated foreign key names and literal values.

\begin{tabular}{|c|c|c|c|}
\hline Task & Level & English Description & SQL \\
\hline $\mathrm{C} 1$ & M & $\begin{array}{l}\text { List all publications in conference } \\
\text { C. }\end{array}$ & $\begin{array}{l}\text { SELECT t2.title FROM conference AS } \mathrm{t} 1 \text { JOIN publication AS t2 ON } \\
\mathrm{t} 1 \text {. cid }=\mathrm{t} 2 \text {. cid WHERE } \mathrm{t} 1 \text {. name }=\text { ' } \mathrm{C} \text { ' }\end{array}$ \\
\hline $\mathrm{C} 2$ & M & List authors in domain D. & $\begin{array}{l}\text { SELECT t1. name FROM author AS t1 JOIN domain_author AS t2 ON t1. aid } \\
=\text { t2. aid JOIN domain AS t3 ON t2.did }=\text { t3.did WHERE } t 3 \text {. name }=\text { ' } D \text { ' }\end{array}$ \\
\hline $\mathrm{C} 3$ & M & $\begin{array}{l}\text { List authors with more than } 5 \text { pa- } \\
\text { pers in conference C. }\end{array}$ & 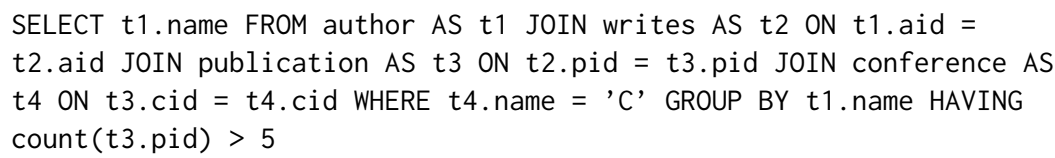 \\
\hline D1 & M & $\begin{array}{l}\text { List the titles of publications pub- } \\
\text { lished by author A. }\end{array}$ & $\begin{array}{l}\text { SELECT t3.title FROM author AS t1 JOIN writes AS t2 ON t1. aid }= \\
\text { t2.aid JOIN publication AS } t 3 \text { ON t2.pid }=\text { t3.pid WHERE t1. name }= \\
\text { ' } A \text { ' }\end{array}$ \\
\hline D2 & M & $\begin{array}{l}\text { List the names of organizations } \\
\text { in continent } C \text {. }\end{array}$ & SELECT name FROM organization WHERE continent $=$ ' $\mathrm{C}$ ' \\
\hline D3 & $\mathrm{H}$ & $\begin{array}{l}\text { List authors with more than } 8 \text { pa- } \\
\text { pers in conference } C \text {. }\end{array}$ & 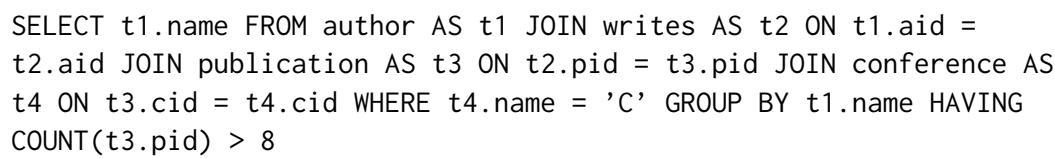 \\
\hline
\end{tabular}

Table 8: Tasks for the user study vs. PBE, with abbreviated foreign key names and literal values. 